\title{
Alveolar Hemorrhage
}

Case 1 A 25-year-old male presented with hemoptysis to the clinic. In BAL, hemorrhage and slightly increased eosinophils were seen, suggestive of vasculitis but also other diseases presenting with alveolar hemorrhage. Therefore, a VATS was done. A $3.2 \times 2.1 \times 1.3 \mathrm{~cm}$ tissue was received.
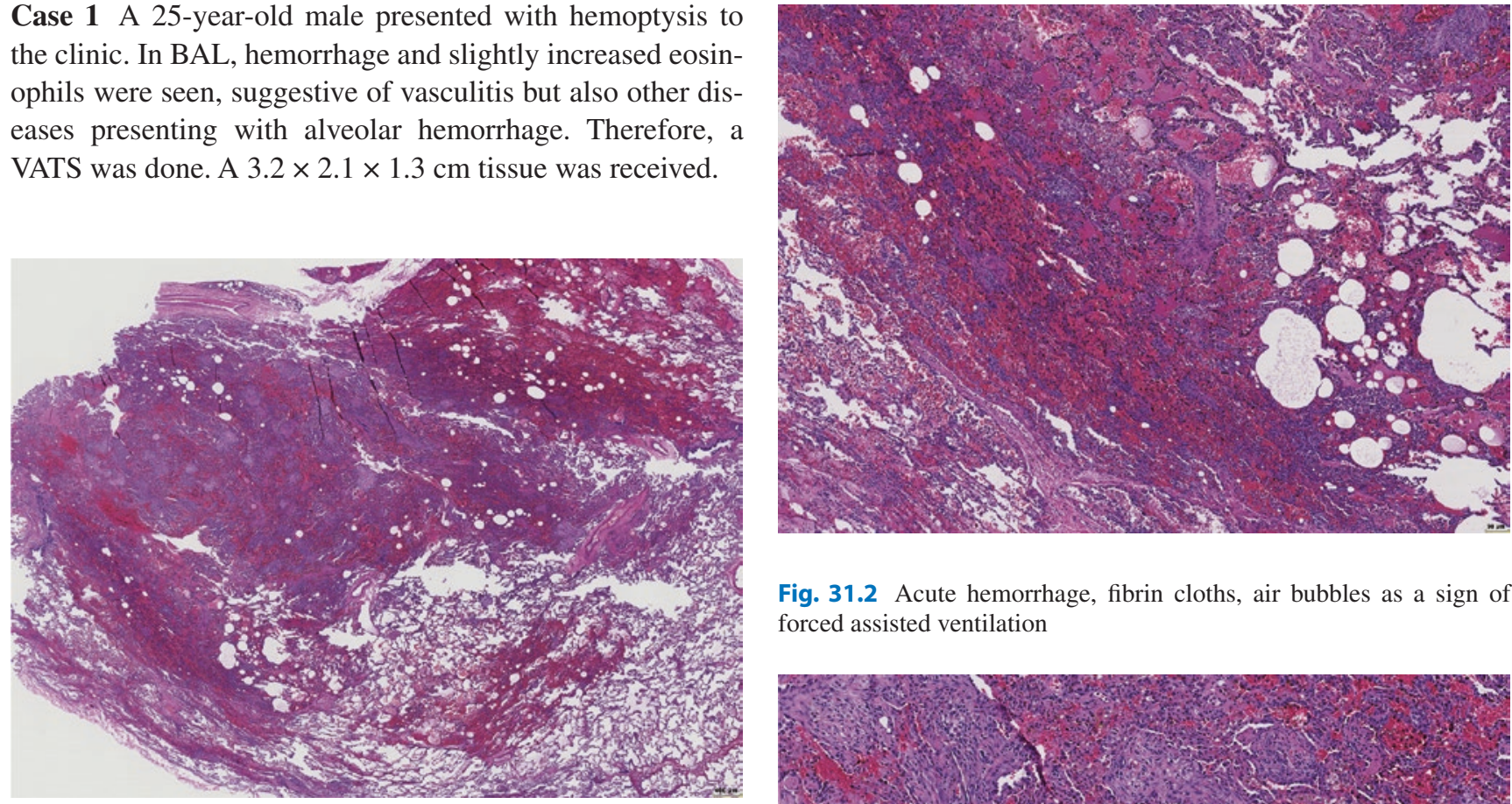

Fig. 31.2 Acute hemorrhage, fibrin cloths, air bubbles as a sign of forced assisted ventilation

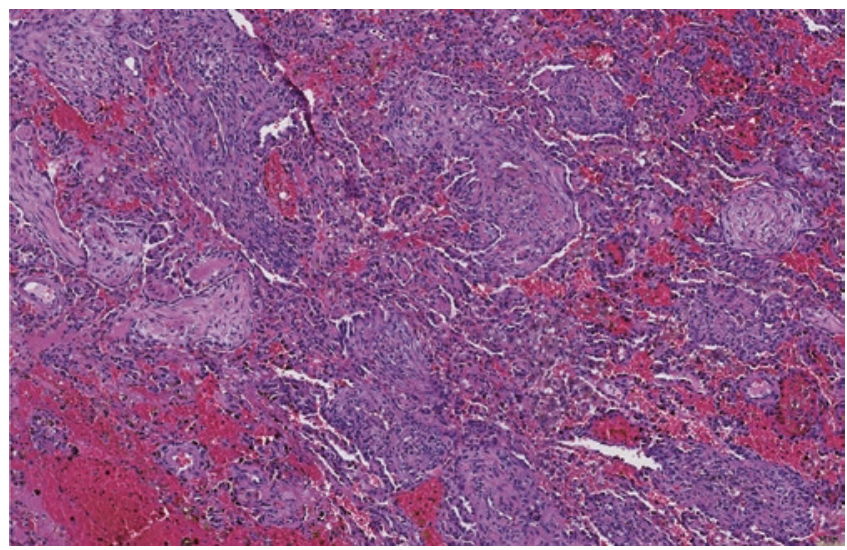

Fig. 31.1 Dense infiltrations in this lung tissue; some more eosinophilic tiny nodules. In addition, there is also hemorrhage

Fig. 31.3 The more eosinophilic nodules are in fact fibrotic areas. Besides fresh hemorrhage, there are also hemosiderin-laden macrophages within alveoli and in the stroma 
Fig. 31.4 Organization of fibrin by granulation tissue, hemosiderin-laden macrophages, and fresh hemorrhage. No inflammation.

Immunohistochemistry for immunoglobulins and complement showed IGG-positivity and complement activation

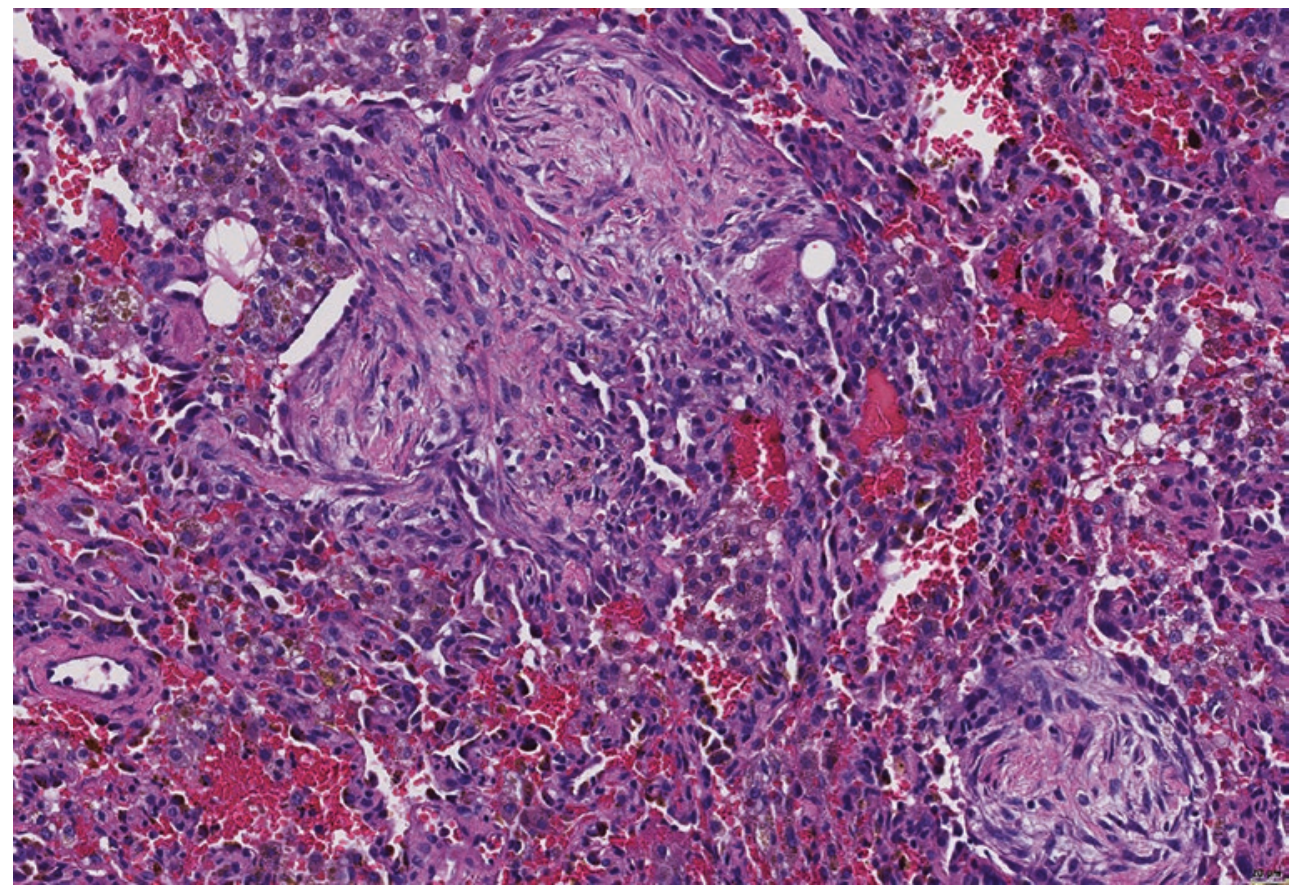

\section{Diagnosis: Goodpasture syndrome.}

Case 2 A 38-year-old man presented with hemoptysis associated with respiratory failure and acute renal failure. Bronchoalveolar lavage (BAL) was consistent with alveolar

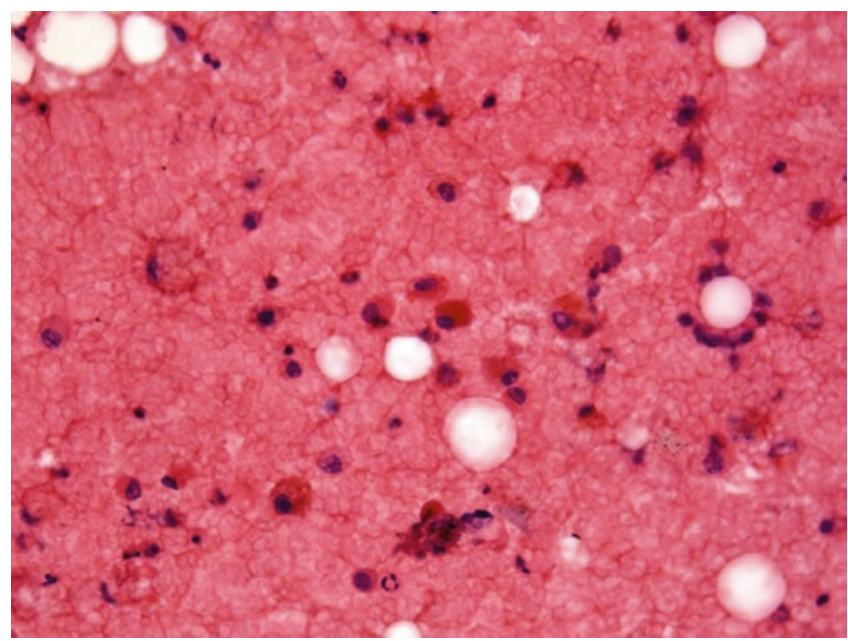

Fig. 31.5 Bronchoalveolar lavage. Blood and macrophages showing pigmented large cytoplasm hemorrhage. Antiglomerular basement membrane antibodies were strongly positive in the serum. The patient died 3 days after admission to the hospital (the slides are from autopsy).

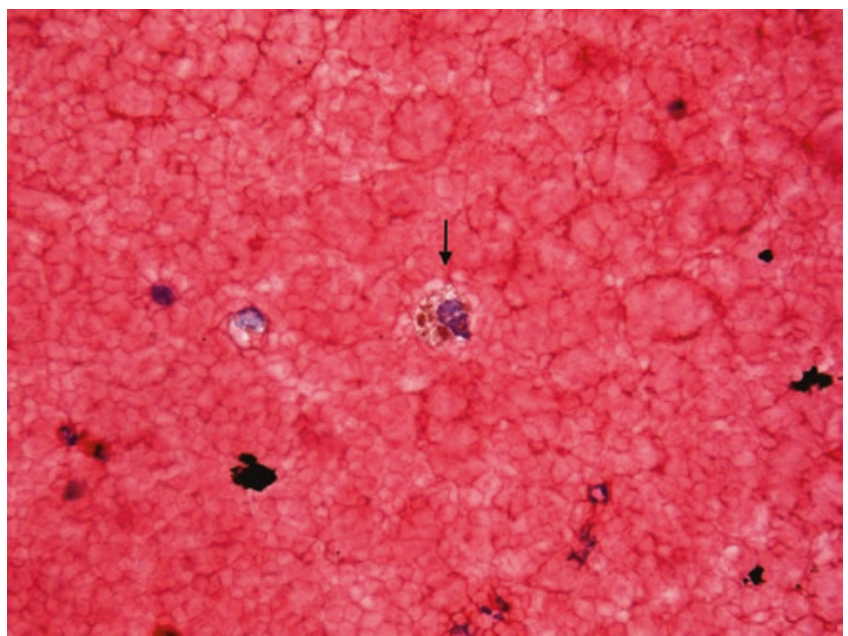

Fig. 31.6 Bronchoalveolar lavage. A few macrophages contain coarse iron pigment (arrow), which is compatible with the clinical diagnosis of Goodpasture Syndrome 


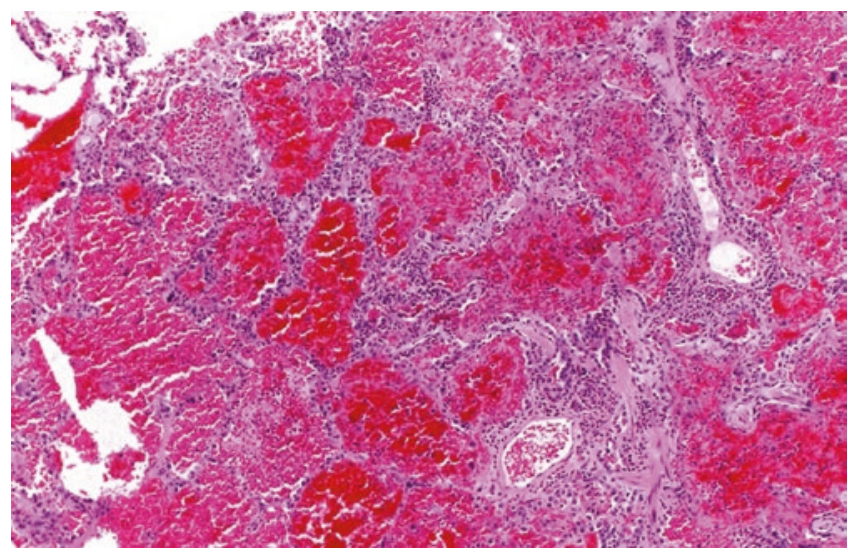

Fig. 31.7 Goodpasture syndrome: The slide shows alveolar spaces filled with blood and fibrin. The interstitium is mildly expanded with inflammatory cells

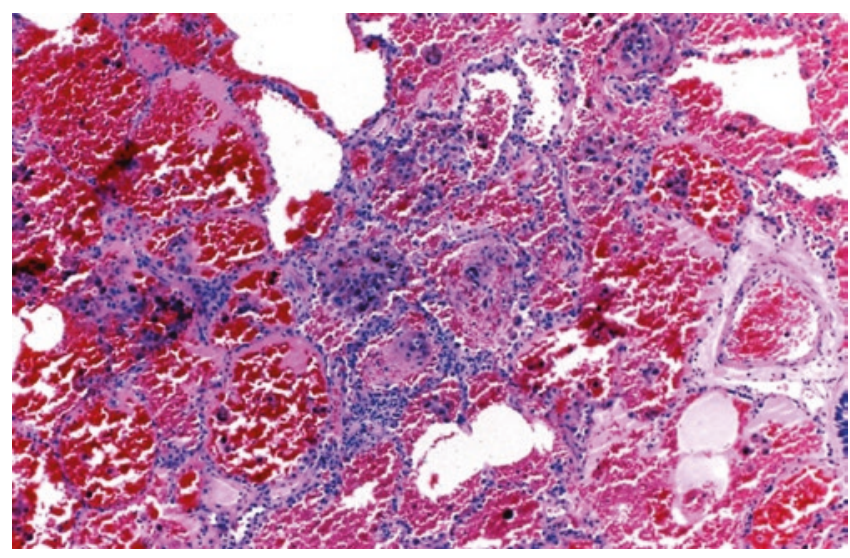

Fig. 31.8 Goodpasture syndrome: The alveolar spaces contain blood, fibrin, and hemosiderin-laden macrophages. Mild inflammatory infiltrate is seen in the alveolar septa

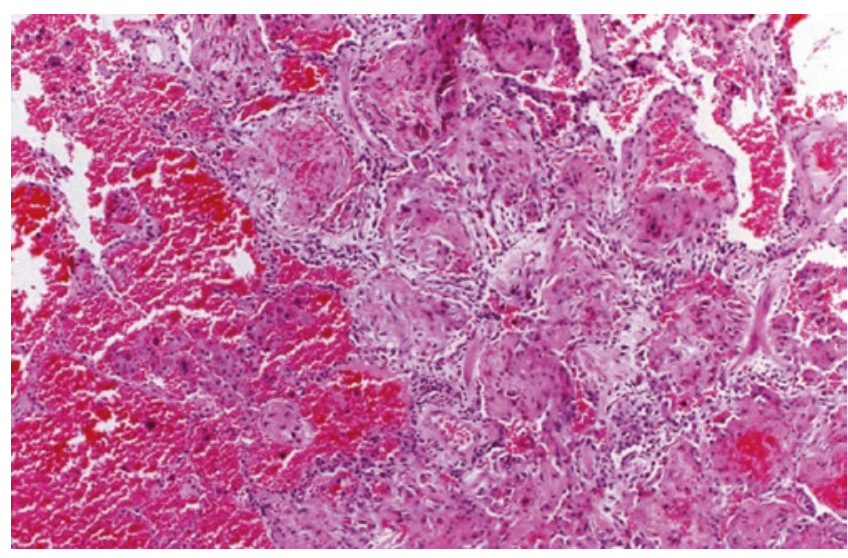

Fig. 31.9 Goodpasture syndrome: Alveolar hemorrhage with a few hemosiderin-laden macrophages and organizing pneumonia-like pattern is seen

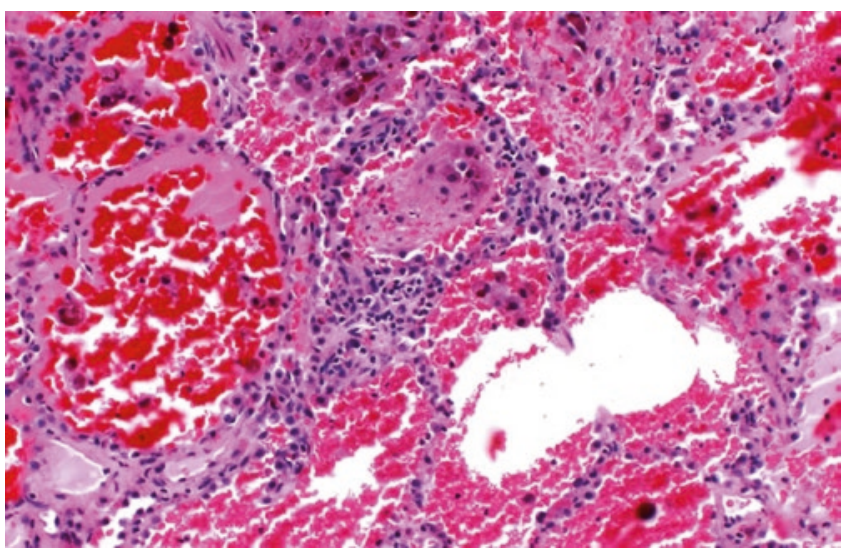

Fig. 31.10 Goodpasture syndrome: At higher magnification, we can appreciate a mild inflammatory infiltrate in the alveolar septa with focal neutrophils

\section{Goodpasture Syndrome (Antiglomerular Basement} Membrane Antibody Disease)

Clinical features

- Most frequently occurs in young patients (20-30 years of age).

- Hemoptysis and anemia are present in about $90 \%$.

- Cough, fever, hematuria, and renal failure are frequently present.

- Circulating anti-GBM are almost always present in the serum.

- Diagnosis can be established by performing a renal biopsy.

Radiologic findings

- Diffuse, bilateral airspaces consolidation or ground glass opacities often with parahilar predominance

Macroscopic findings

- Dense, firm, red lungs

Microscopic findings

- Extensive intra-alveolar hemorrhage with accumulation of red blood cells and hemosiderin-laden macrophages in alveolar spaces.

- Some cases show hyaline membranes in addition to alveolar hemorrhage.

- Nonspecific thickening of alveolar septa may be seen.

- Capillaritis and small vessel vasculitis is very rare and only focal. 
- Immunofluorescence/immunohistochemistry reveals linear staining for immunoglobulins (usually $\operatorname{IgG}$ ) and complement along basement membranes of capillaries and the alveolar septa

Differential diagnosis

- Other causes of pulmonary capillaritis

- DAH associated with other processes

Prognosis and therapy

- Plasmapheresis, corticosteroids, cyclophosphamide, and azathioprine.

- The 2-year survival rate is approximately $50 \%$

Case 3 A 36-year-old female with a well-known history of systemic lupus erythematosus (SLE). She presented with fever and hemoptysis. CT scan showed diffuse pulmonary infiltrates.

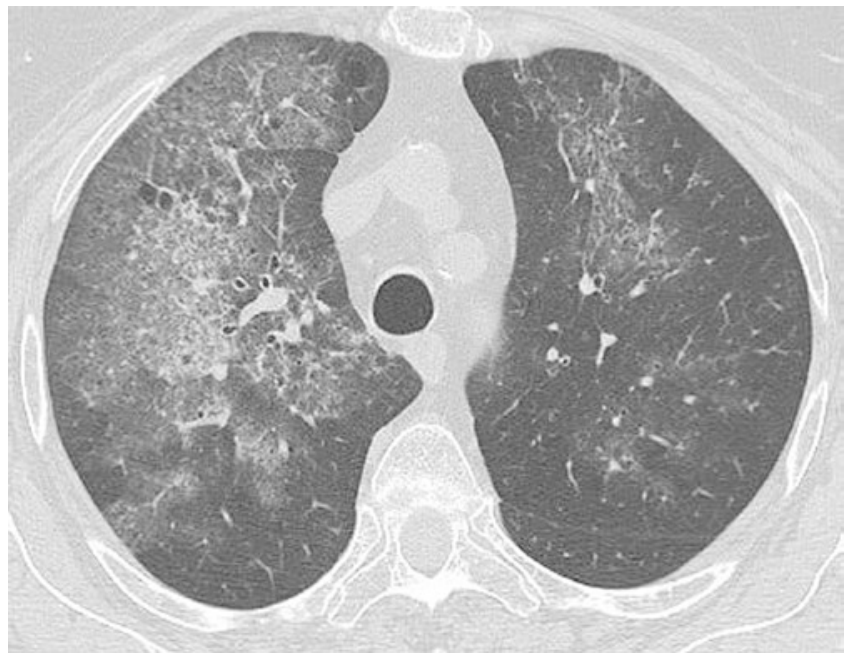

Fig. 31.11 Acute Lupus Pneumonia: CT scan showing diffuse pulmonary infiltrates with a ground glass appearance

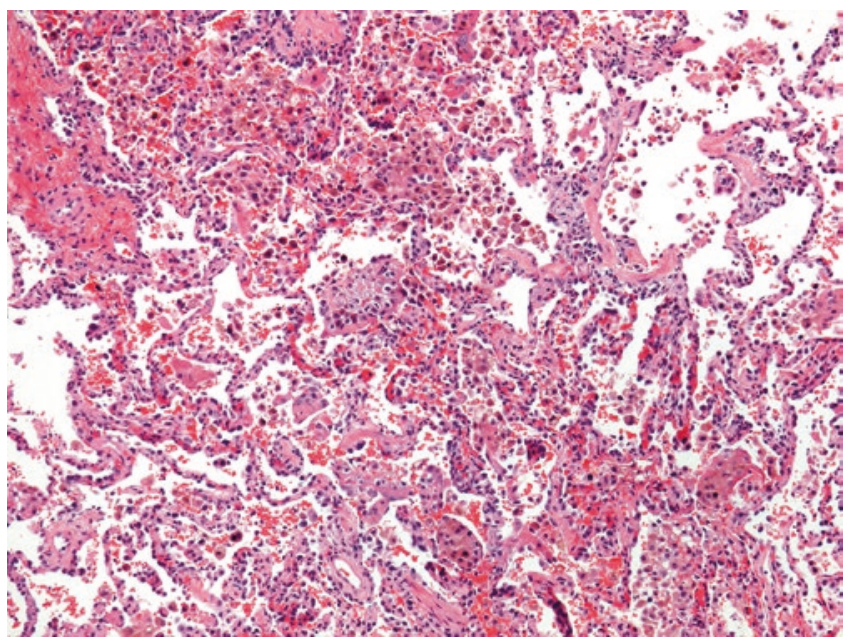

Fig. 31.12 Acute Lupus Pneumonia: The alveolar spaces are filled by numerous hemosiderin-laden macrophages, erythrocytes, and fibrin. This combination is referred to a non recent, bland alveolar hemorrhage

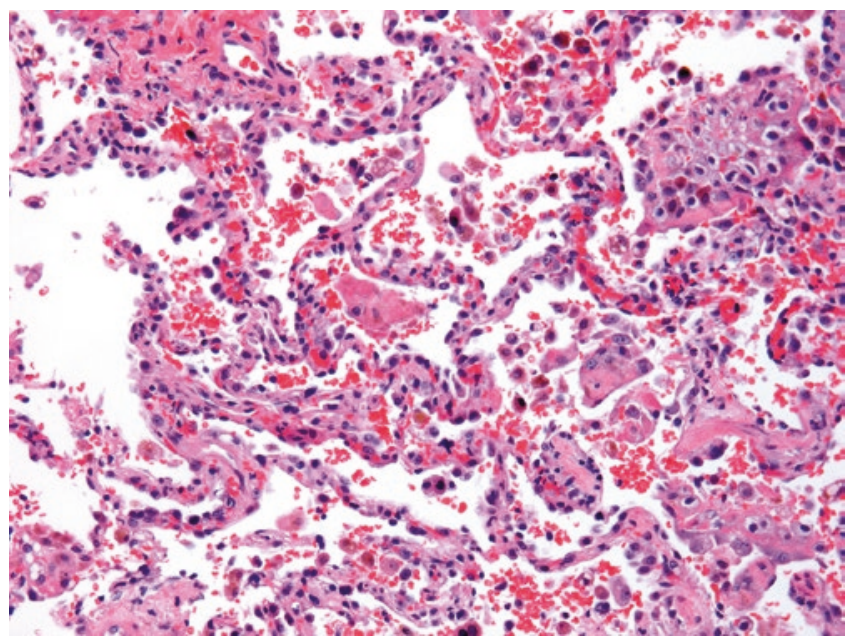

Fig. 31.13 Acute Lupus Pneumonia: Higher magnification showing type 2 pneumocytes hyperplasia and focal neutrophil accumulation in capillaries

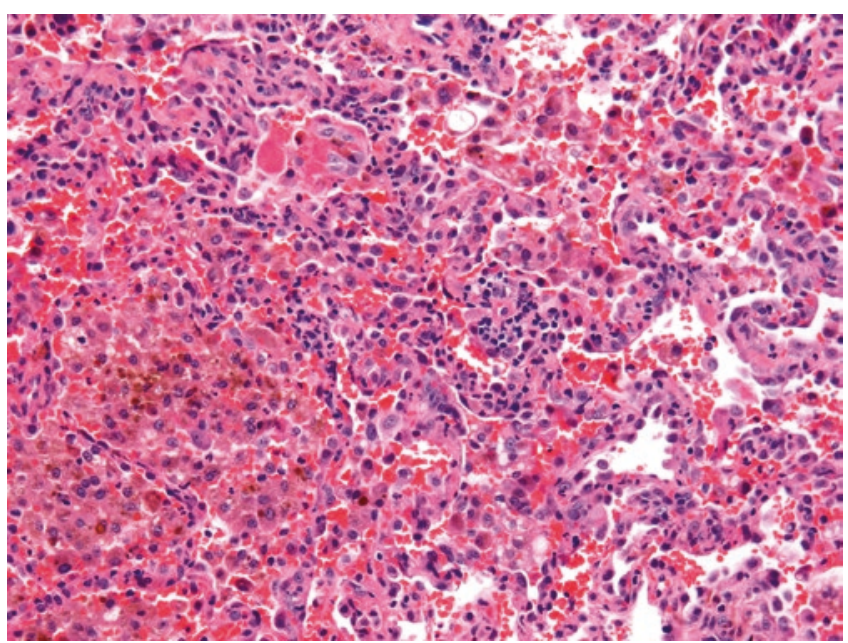

Fig. 31.14 Acute Lupus Pneumonia: Acute inflammation is seen in alveolar septa, indicating capillaritis 


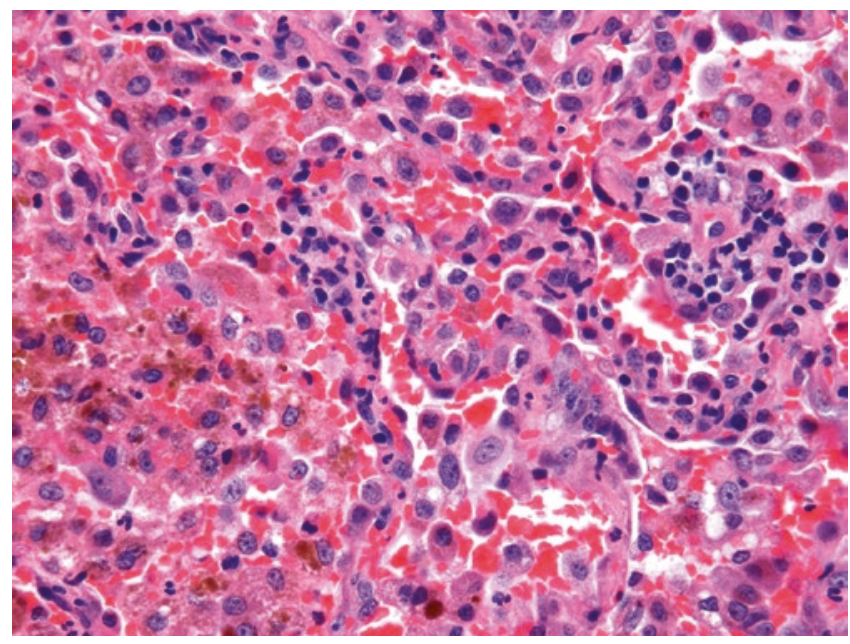

Fig. 31.15 Acute Lupus Pneumonia: Higher magnification emphasizes the presence of capillaritis that is commonly present in cases of SLE-induced hemorrhage

Case 4 A 77-year-old male presented with hemoptysis. There was a history of hypertensive renal disease with renal insufficiency. ANCA tests were negative, no other organs were affected. On CT scan, many ground glass opacities were seen. Slides and tissue blocks were submitted for consultation from the VATS.

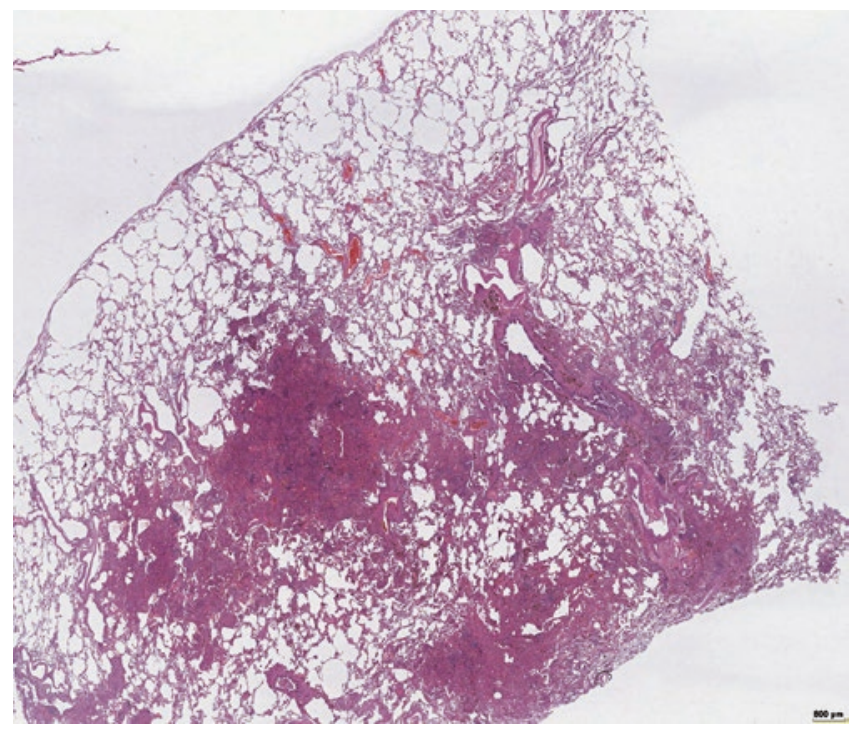

Fig. 31.16 Overview of lung tissue with focal densities in the lung periphery

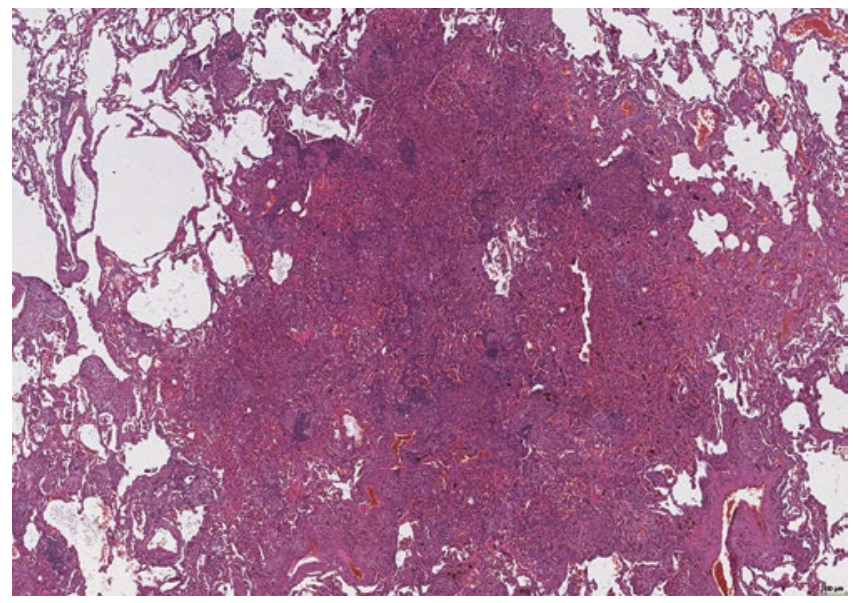

Fig. 31.17 Alveolar hemorrhage and focal inflammation

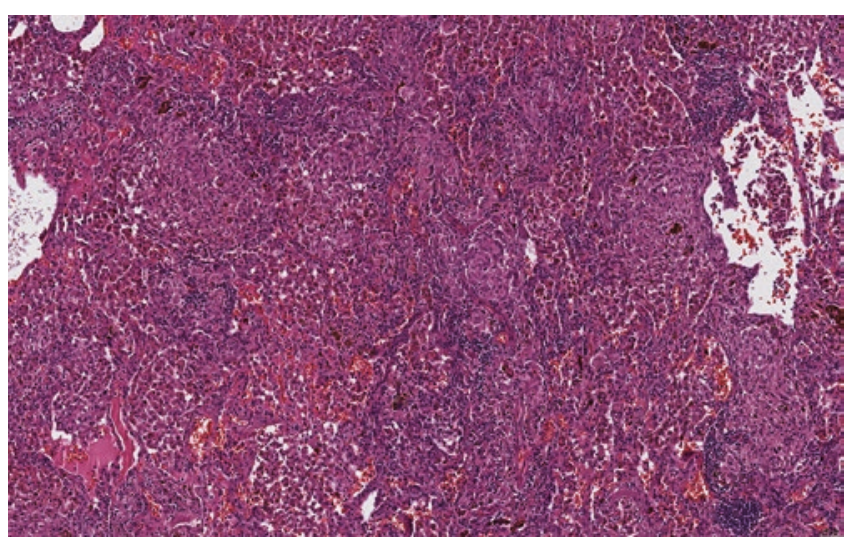

Fig. 31.18 Alveolar hemorrhage acute and old; many hemosiderinladen macrophages and organization of the injury by granulation tissue

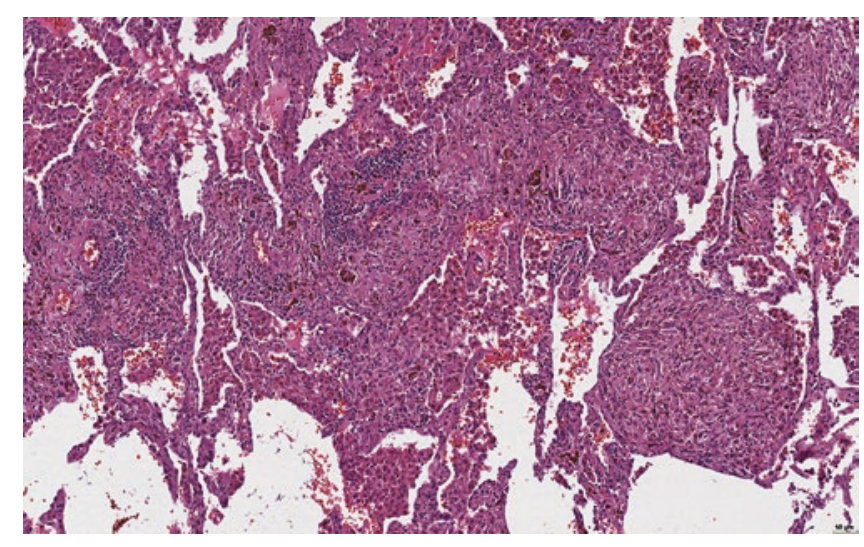

Fig. 31.19 Nodules of granulation tissue with some hemosiderinladen macrophages. In addition, fresh hemorrhage and focal lymphocytic infiltration, especially perivascular 
Fig. 31.20 High-power view of granulation tissue and macrophages intra-alveolar and interstitial, including hemosiderin containing macrophages

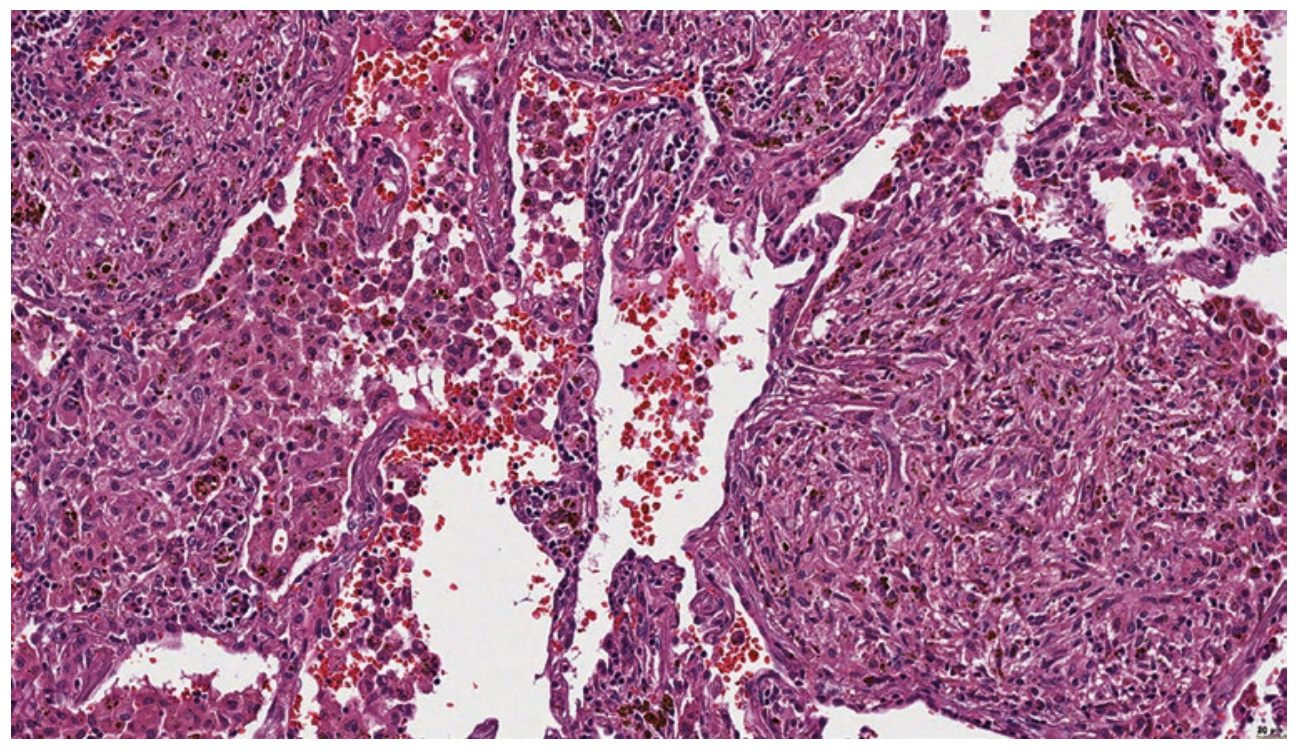

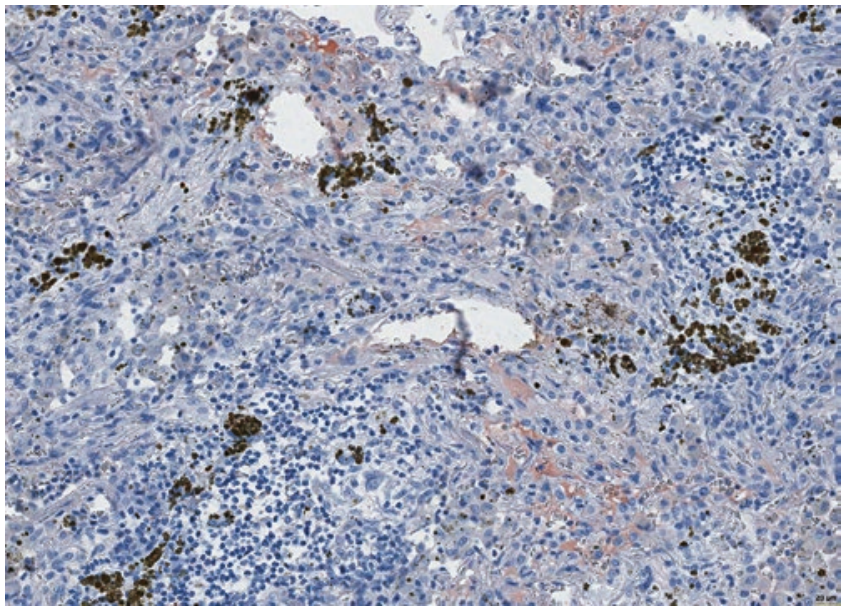

Fig. 31.21 Focal positivity for complement component 3C, pointing to an immune mechanism

Diagnosis: Autoimmune disease with recurrent hemorrhage, organization, lymphocytic infiltrates, and immune complex deposition with complement activation; all together would favor systemic lupus erythema-

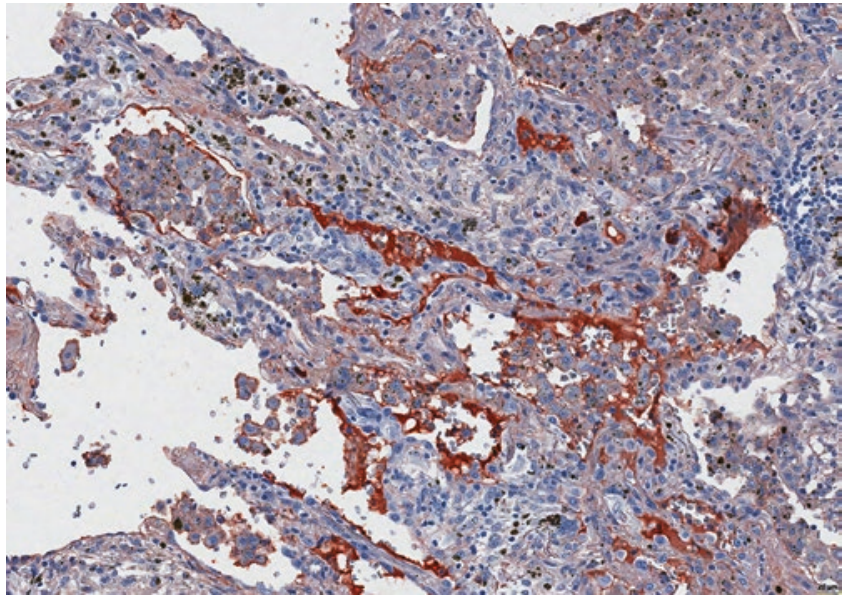

Fig. 31.22 Deposits of immune complexes positive for $\mathrm{IgG}$

todes. After submission of the suggested diagnosis, antinuclear antibodies were found and SLE was confirmed, clinically. 
Case 5 A 50-year-old obese lady, 13 pack-year cigarette smoker. She kept pet parrots at home, had episodes of hemoptysis during and after the contact with the animals. History of asthma, sinusitis; gastrointestinal complaints, diverticulosis, atrophic gastritis; elevated IgE, no peripheral eosinophilia. ANA, ANCA rheumatoid factor, serum antiGBM, and avian precipitins were negative. Steroid medication 3 weeks prior to open lung biopsy.

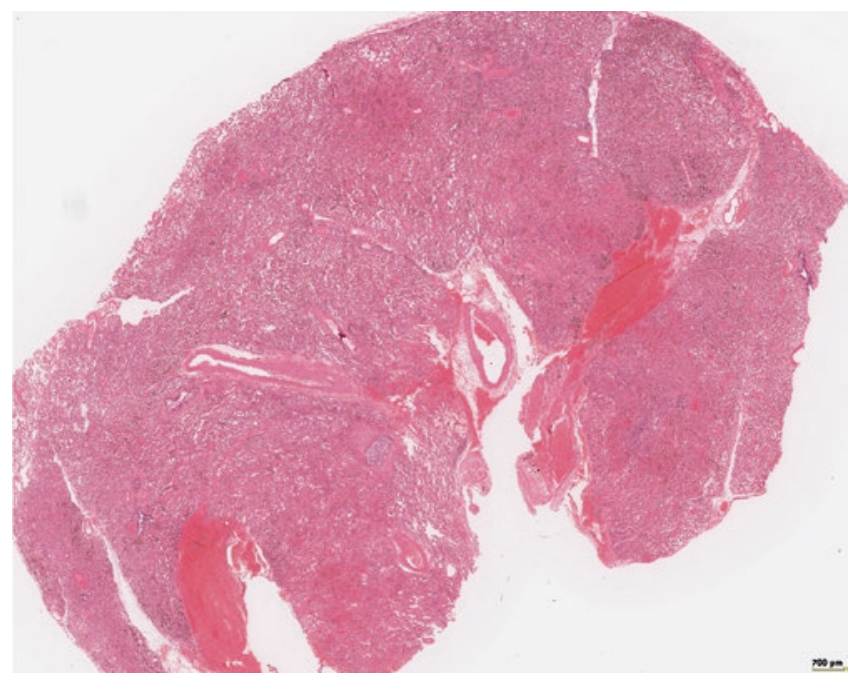

Fig. 31.23 Lung tissue with alveolar hemorrhage

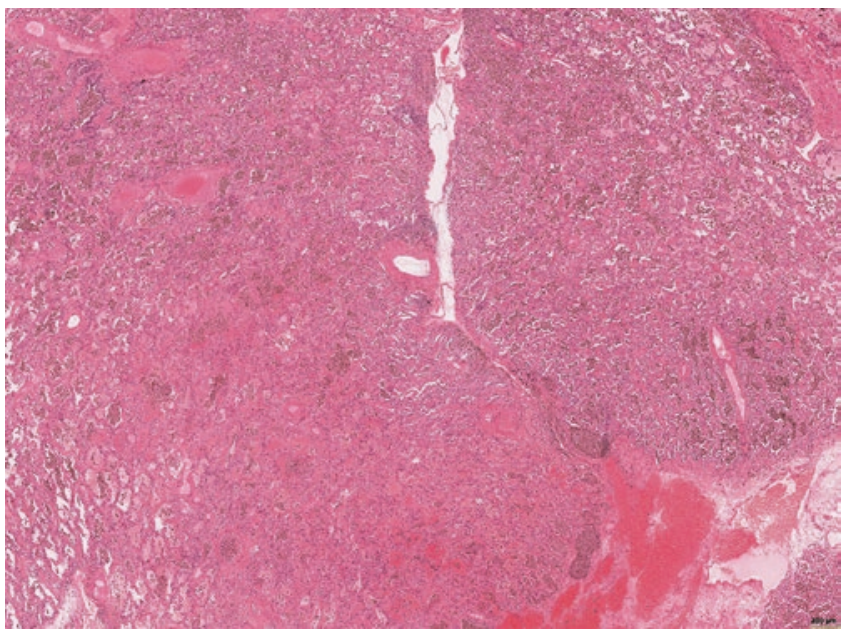

Fig. 31.24 Acute hemorrhage and macrophages with hemosiderin

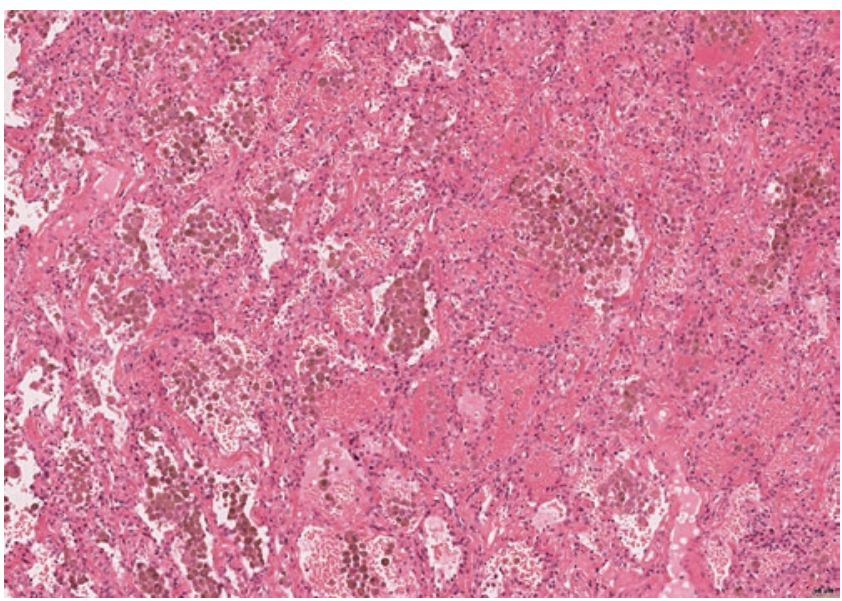

Fig. 31.25 Macrophages with hemosiderin and fresh hemorrhage; no inflammatory infiltration

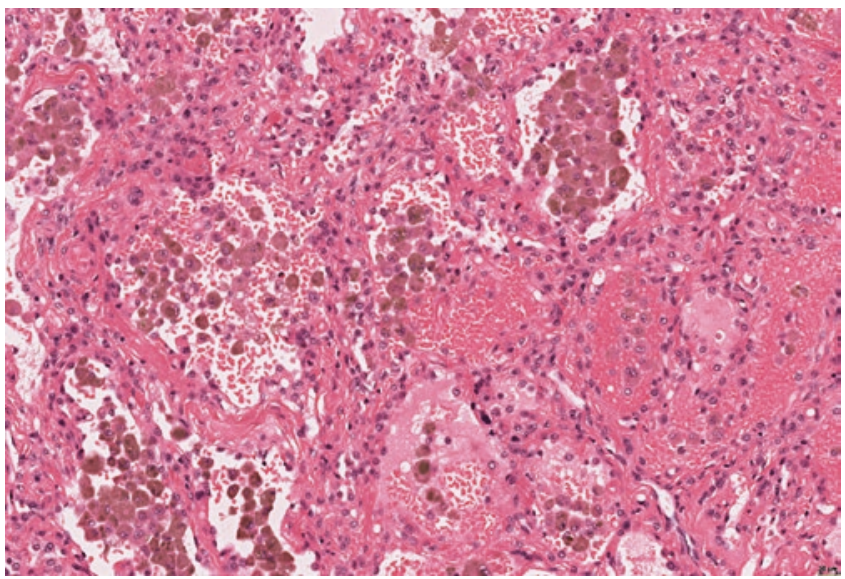

Fig. 31.26 Recurrent hemorrhage, no other morphologic changes are visible

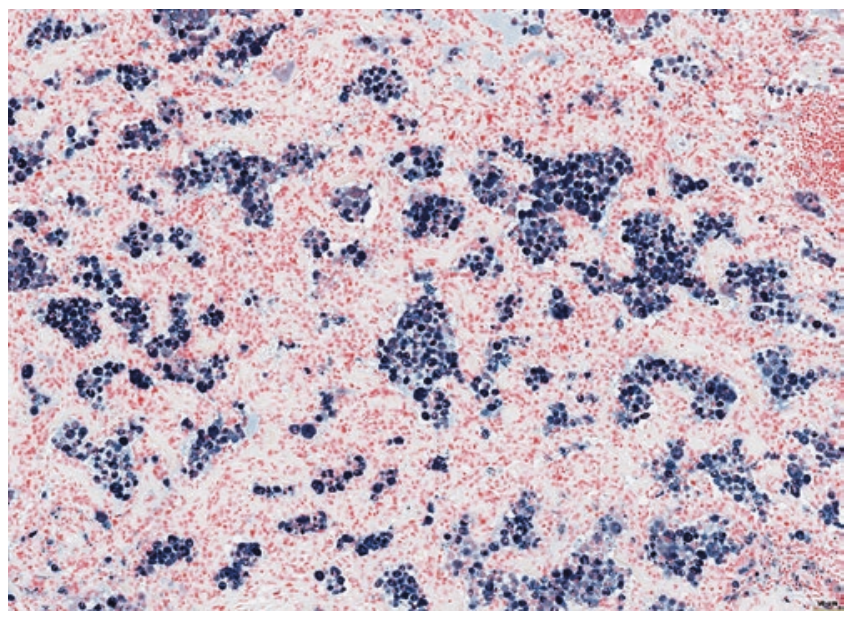

Fig. 31.27 Hemosiderin-laden macrophages demonstrated by Prussian blue stain. By immunohistochemistry, no autoantibodies could be demonstrated, ruling out Goodpasture syndrome. No immune cell infiltrations, ruling out autoimmune diseases

Diagnosis: Alveolar hemorrhage syndrome of unknown cause. Clinically an antiphospholipid antibody syndrome was finally diagnosed. 
Case 6 A 23-year-old female presented with myalgias, hemoptysis, and diffuse pulmonary infiltrates. She had a positive c-ANCA test result. Lung biopsy.

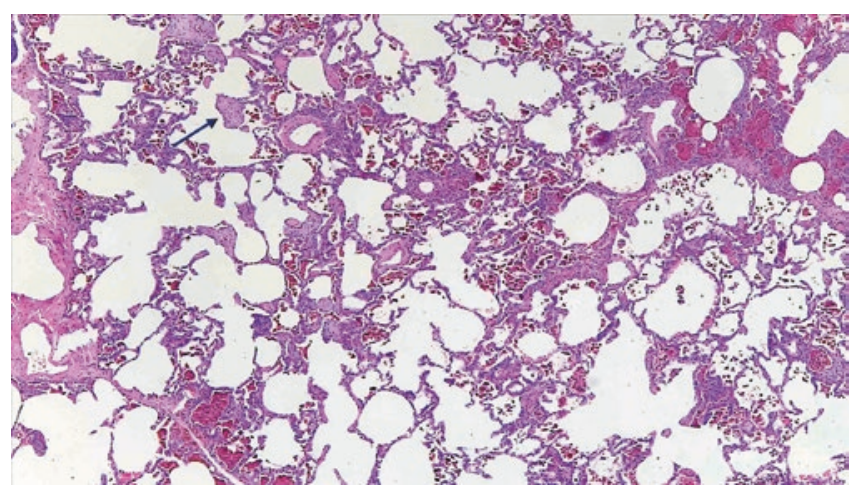

Fig. 31.28 Alveolar hemorrhage (capillaritis): There is a diffuse intraalveolar accumulation of hemosiderin-filled macrophages with plugs of fibroblastic tissue (organizing pneumonia) (arrows)

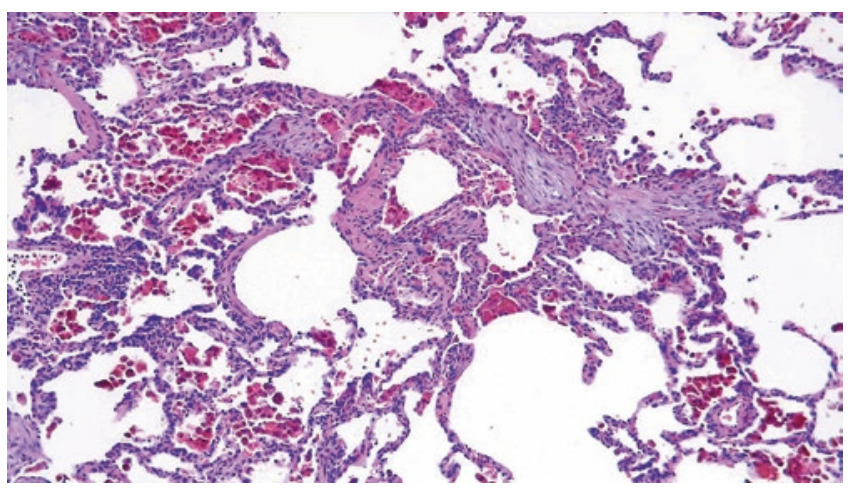

Fig. 31.29 Alveolar hemorrhage (capillaritis): Higher magnification showing organization (organizing pneumonia pattern) and intraalveolar accumulation of hemosiderin-filled macrophages. There are a few interstitial inflammatory cells

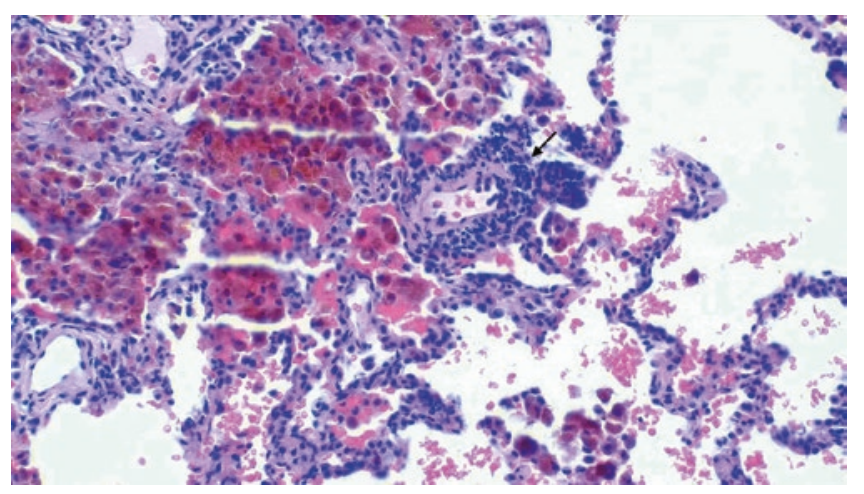

Fig. 31.30 Alveolar hemorrhage (capillaritis): The alveolar hemosiderosis is associated with capillaritis. The morphologic pattern of this case characterized by old hemorrhage and capillaritis is not specific and can be observed in Lupus, early Wegener's granulomatosis, and microscopic polyangiitis

\section{Alveolar Hemorrhage in Autoimmune Disease}

In some autoimmune diseases (AIDs) such as lupus, alveolar hemorrhage is common and sometimes the only presentation. It is caused by autoantibodies or immune complexes damaging the endothelium of small blood vessels, or due to vasculitis. The AIDs usually presenting with hemorrhage are systemic Lupus, systemic sclerosis, primary pulmonary vasculitides, antiphospholipid autoantibodies, Goodpasture syndrome, and idiopathic pulmonary hemosiderosis. There are some rare AIDs, which can also present with hemorrhage, such as Behcèt disease and autoimmune hemolytic anemia with lung involvement.

\section{Clinical and radiological presentation}

- Irregular ground glass opacities in both lungs

Histology

- Alveolar hemorrhage

- Vasculitis of small blood vessels or immune complex deposits with inflammatory reaction

\section{Therapy}

- Corticosteroid treatment Immunosuppressive therapy

Case 7 A 75-year-old healthy man with a history of fever and fatigue of a week duration interpreted as a flu syndrome presented with an acute respiratory distress and hemoptysis. CT scan revealed bilateral ground glass opacities. He died 2 days after the admission to the hospital. Autopsy was done. The lung parenchyma is from the autopsy.

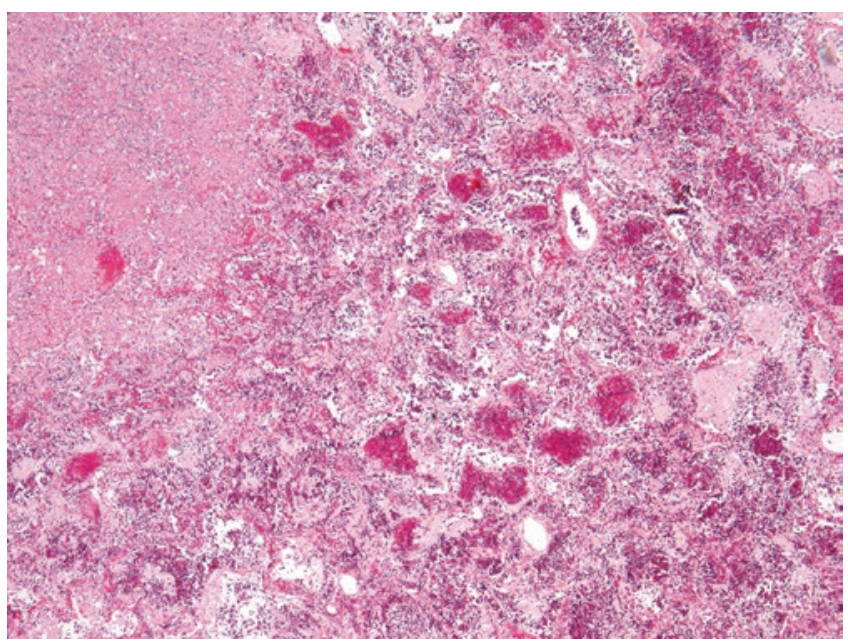

Fig. 31.31 Intravascular large B-cell lymphoma. At low magnification, the alveolar spaces are filled by blood and fibrin mixed with necrotic material. The alveolar septa are slightly enlarged 
Fig. 31.32 Intravascular large B-cell lymphoma. In this field, the alveolar spaces contain fibrin and blood. The pulmonary capillaries contain lymphoid cells with large nuclei. A marginal necrotic area is also present
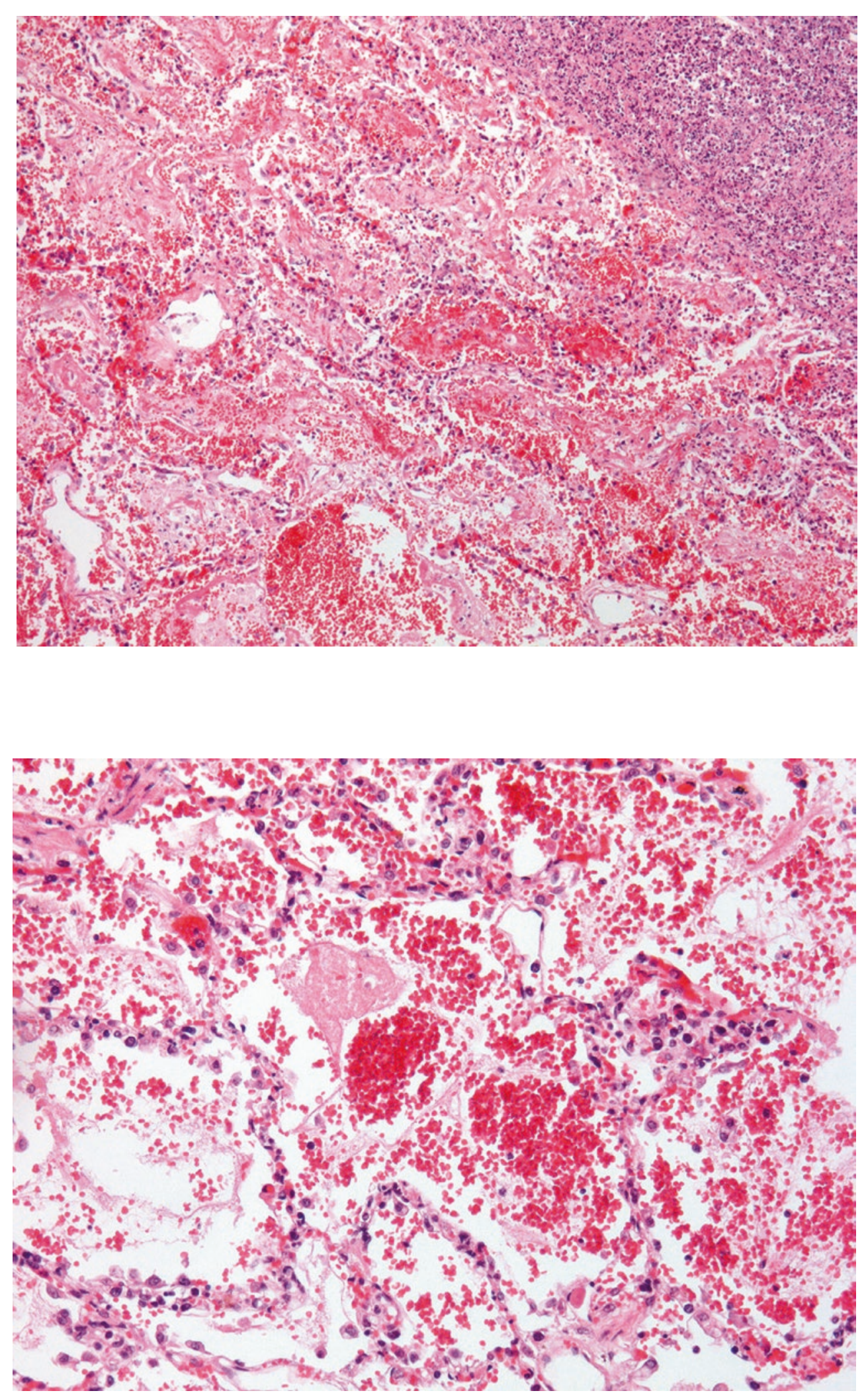

Fig. 31.33 Intravascular large B-cell lymphoma. At higher-power view capillaries and arterioles contain atypical lymphoid cells with large vesicular nuclei 


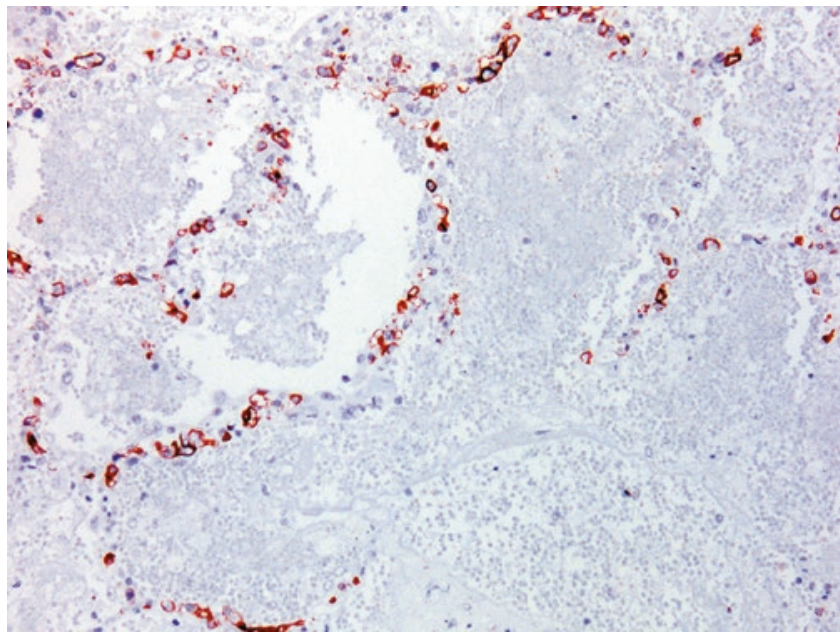

Fig. 31.34 Intravascular large B-cell lymphoma. The intravascular lymphoid cells have a B-cell immunophenotype and express CD20

Intravascular large B-cell lymphoma is a systemic disease that involves multiple organs and usually presents with central nervous system or cutaneous manifestations. It frequently involves the small pulmonary vessels and rarely presents with respiratory symptoms (if present it can simulate infection with fever). Microscopically, small pulmonary blood vessels are occluded by large atypical lymphoid cells.

Case 8 A 68-year-old female with a long history of mitral stenosis treated with valvuloplasty. The slide related to lung parenchyma is from the autopsy.

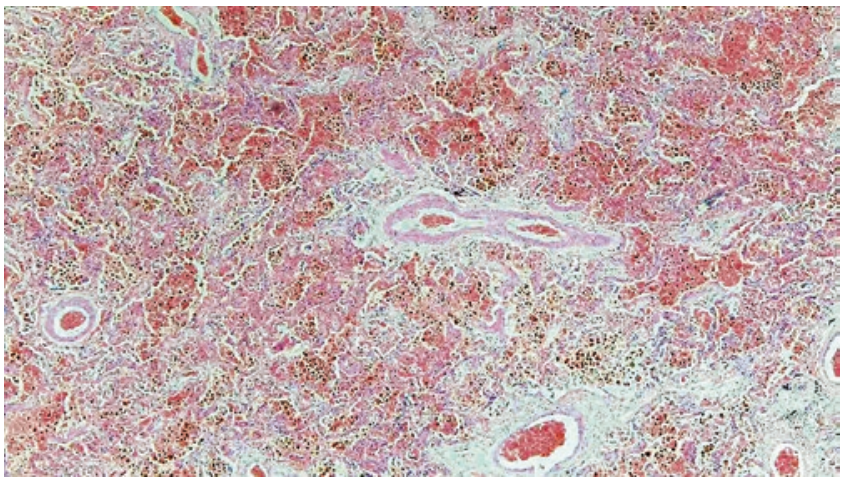

Fig. 31.35 Chronic venous congestion. The alveolar spaces contain blood and numerous hemosiderin-laden macrophages

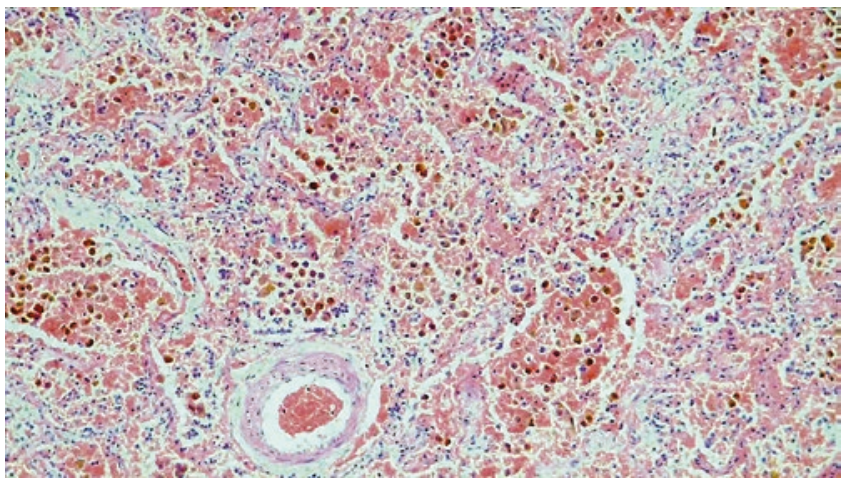

Fig. 31.36 Chronic venous congestion. The alveolar spaces filled with red cells, fibrin, and hemosiderin-laden macrophages are associated with a mild thickening of the alveolar septa and mild thickening of the media of the artery wall

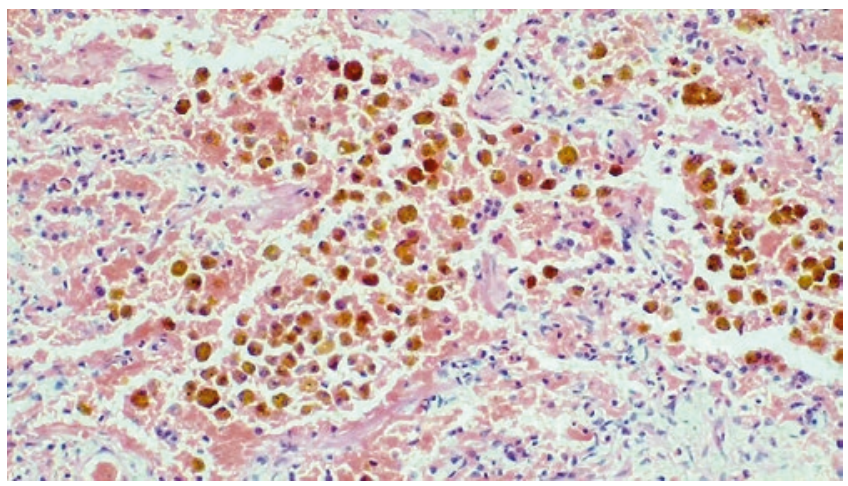

Fig. 31.37 Chronic venous congestion. Numerous macrophages with coarse iron pigment mixed with fibrin

Case 9 A 53-year-old man, smoker, who underwent resection of pulmonary hamartoma. Two pieces of lung tissue were sent to the laboratory, one containing a nodular chondroid hamartoma and one composed of "normal" lung parenchyma that is presented here.

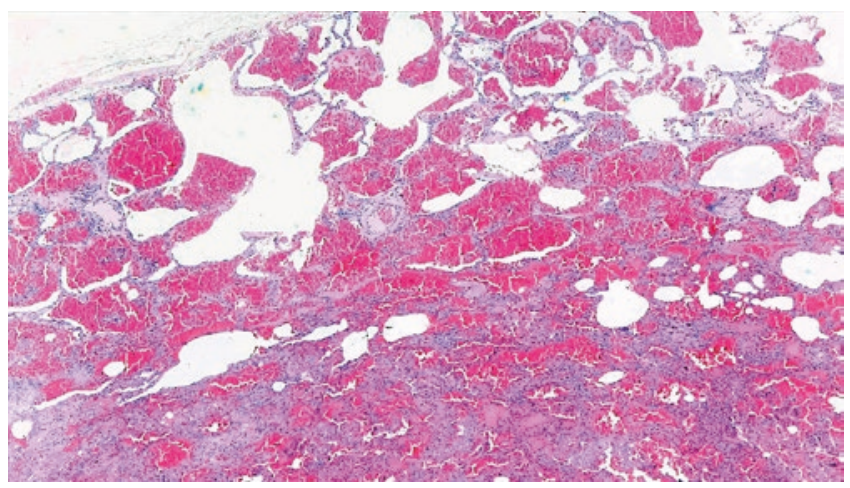

Fig. 31.38 Artifactual intra-alveolar hemorrhage. The lung parenchyma shows a mild interstitial fibrosis and diffuse intra-alveolar hemorrhage 


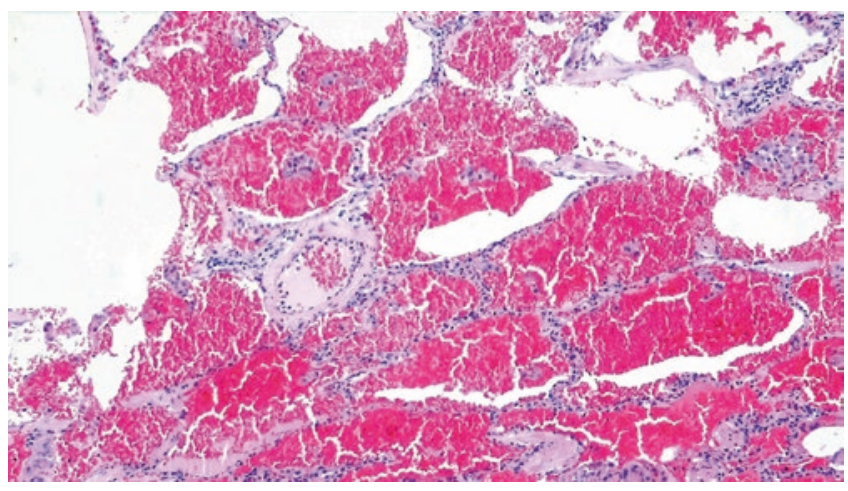

Fig. 31.39 Artifactual intra-alveolar hemorrhage. The diffuse intra-alveolar hemorrhage results from the filling of the alveolar spaces with fresh blood. No hemosiderin-laden macrophages are seen. The intra-alveolar hemorrhage associated with hemosiderin-laden macrophages would have indicated that bleeding occurred in the past

Case 10 An 8-year-old girl with known diffuse lung disease and iron-deficiency anemia for 6 years. Circulating antiGMB antibodies were negative. She was treated with corticosteroids. Sudden death following hemoptysis. Autopsy: large and dark lungs.

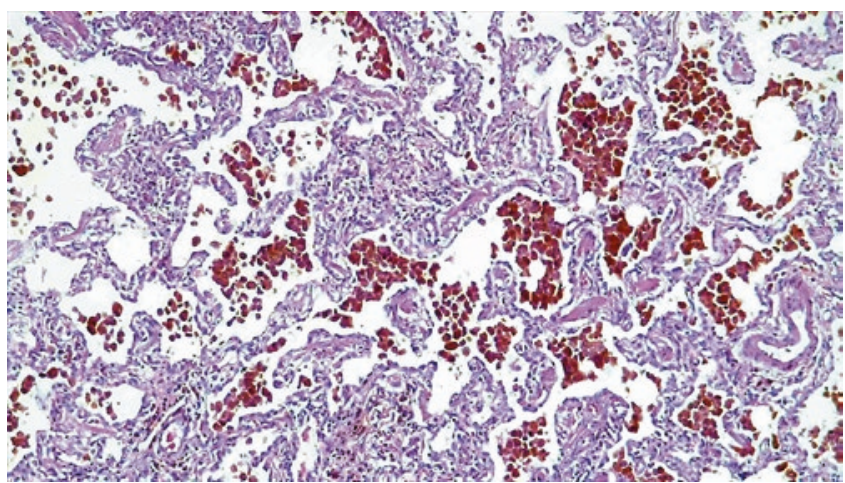

Fig. 31.40 Idiopathic pulmonary hemosiderosis: There is prominent accumulation of hemosiderin-filled macrophages in airspaces with a discrete thickening of the alveolar septa reminiscent of desquamative interstitial pneumonia

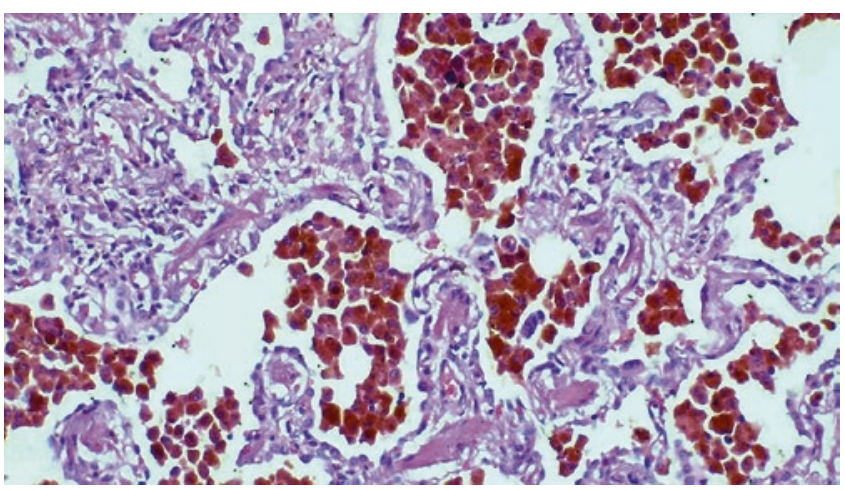

Fig. 31.41 Idiopathic pulmonary hemosiderosis: The higher-power view emphasizes the intra-alveolar accumulation of hemosiderin-filled macrophages and the nonspecific interstitial fibrosis with type 2 hyperplasia

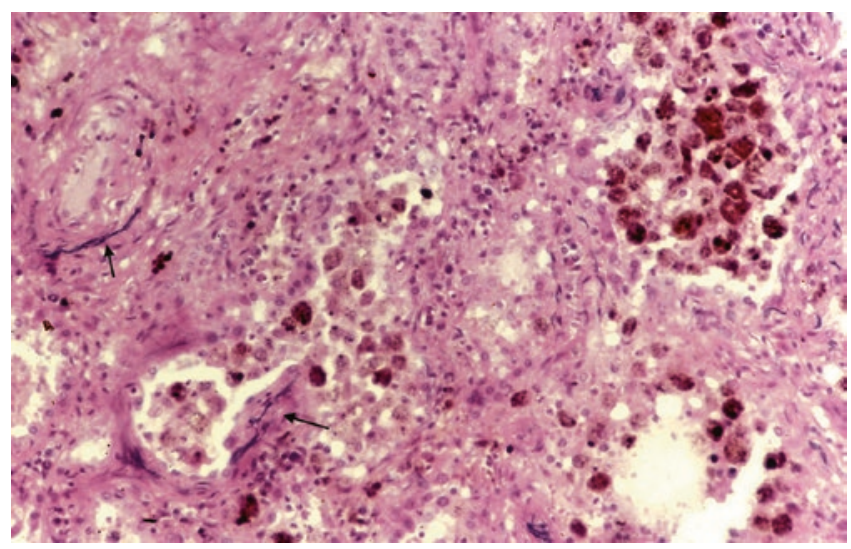

Fig. 31.42 Idiopathic pulmonary hemosiderosis: In this field, there is an encrustation of vascular elastic tissue by iron (arrows). This is a common finding in idiopathic hemosiderosis, but it is not specific for this entity

Idiopathic Pulmonary Hemosiderosis (IPH)

Clinical features

- Rare disease.

- Equal distribution between male and female.

- Most cases occur in children under 10 years old.

- A few cases may present in adults with a male predominance.

- Familial cases have been described.

- Recurrent episodes of alveolar hemorrhage often with hemoptysis.

- Cough, dyspnea, and iron-deficiency anemia are frequently present.

- IHP is sometimes associated with celiac disease

Radiologic findings

- Diffuse, bilateral airspaces consolidation or ground glass opacities

Macroscopic findings

- Dense, firm, brown lungs

Microscopic findings

- Extensive intra-alveolar hemorrhage

- Chronic changes with hemosiderin and mild interstitial widening

- Minimal inflammation

- No capillaritis

Differential diagnosis

- Other causes of pulmonary hemorrhage

Prognosis and therapy

- Variable response to corticosteroids

- Median survival of 3-5 years 